\title{
Apreciaciones de trabajadores de la salud sobre la implementación del sistema de seguridad en la utilización de medicamentos
}

Assessments of health workers on the implementation of the safety system in the use of medications

\author{
Aleyda Restrepo-Vásquez ${ }^{1}$ orcid.org/0000-0002-7573-9956 \\ Julia Inés Escobar-Montoya ${ }^{*}$ orcid.org/0000-0002-8523-9760
}

1 Facultad de Ciencias de la Salud, Universidad Libre Seccional Pereira. Pereira, Colombia

Fecha de recepción: Marzo 16 - 2016

Fecha de revisión: Febrero 24 - 2017

Fecha de aceptación: Marzo 24 - 2017

Restrepo-Vásquez A, Escobar-Montoya JI. Apreciaciones de trabajadores de la salud sobre la implementación del sistema de seguridad en la utilización de medicamentos. Univ. Salud. 2017;19(1):7-16. DOI: http://dx.doi.org/10.22267/rus.171901.64

\begin{abstract}
Resumen
Introducción: Los errores en la medicación es uno de los eventos adversos más frecuentes en la mayoría de los países de América Latina, lo que ha llevado a promover un sistema para su identificación y reducción en las instituciones de salud. Objetivo: Determinar las apreciaciones del personal de la salud sobre el grado de implementación del sistema de seguridad del paciente en la utilización de medicamentos. Materiales y métodos: Estudio cuantitativo descriptivo en una población de 73 trabajadores de la salud. Se diseñó una encuesta a partir de dos instrumentos: el cuestionario de autoevaluación de la seguridad del sistema de utilización de los medicamentos en los hospitales y el cuestionario sobre seguridad de los pacientes: versión española del Hospital del Ministerio de Sanidad y Consumo y se contrastaron con los lineamientos de la política de seguridad del paciente para Colombia. Las respuestas se midieron mediante un baremo con cuatro categorías: cumple, cumple medianamente, no cumple y no sabe. Resultados: El porcentaje promedio de personas que manifestaron un total cumplimiento en la aplicación de la política fue de 65,1\% en la estandarización, almacenamiento y distribución de los medicamentos, 61,7\% en su prescripción y 65,5\% en su administración y registro. Conclusiones: Se evidenció que aproximadamente la mitad de los encuestados apreciaban falta de cumplimiento en la implementación del sistema de seguridad del paciente que enmarca los procesos de utilización de medicamentos en todas sus etapas. Lo anterior deja ver que, si bien existe teóricamente un sistema de seguridad en la medicación de los pacientes en las instituciones estudiadas, es necesario que se fortalezcan las acciones gerenciales tendientes a garantizar su aplicación efectiva con todos los actores implicados, siendo la vigilancia permanente de los procesos un pilar fundamental.
\end{abstract}

Palabras clave: Seguridad del paciente; utilización de medicamentos; daño del paciente; errores de medicación; servicios de salud. (Fuente: DeCS, Bireme).

\begin{abstract}
Introduction: Errors in medication is one of the most frequent adverse events in most of the countries in Latin America, which has led to promote a system for its identification and reduction in health institutions. Objective: To determine the assessments of health personnel on the degree of implementation of the system of patient safety in the use of medicines. Materials and methods: A quantitative descriptive study with a population of 73 health workers was conducted. A survey was designed based on two instruments: the self-assessment questionnaire for the security of the system for the use of medicines in hospitals and the questionnaire on patient safety: Spanish version of the Hospital of the Ministry of Health and Consumption, and they were contrasted with the guidelines of the
\end{abstract}

*Autor de correspondencia

Julia Inés Escobar Montoya

e-mail: jiescobar@unilibrepereira.edu.co 
patient security policy for Colombia. The answers were measured by a scale with four categories: strongly agree, agree, disagree and do not know. Results: The average percentage of people who indicated a total compliance in the implementation of the policy in the standardization was of $65.1 \%$, storage and distribution of medicines in its prescription $61.7 \%$, and $65.5 \%$ in its administration and registration. Conclusions: It was evidenced that approximately half the respondents appreciated lack of compliance in the implementation of the system of patient safety that frames the processes of drug use in all its stages. The foregoing reveals that while there is a security system in the patients' medication in the institutions studied in theory, there is a need to strengthen the management actions aimed at ensuring its effective implementation with all actors involved, being the permanent monitoring of the processes a fundamental pillar.

Keywords: Patient safety; drug utilization; patient harm; medication errors; health services. (Source: DeCS, Bireme).

\section{Introducción}

El actual movimiento mundial de seguridad del paciente alcanzó relevancia cuando en 1999 el Instituto de Medicina de la Academia Nacional de Ciencias de los Estados Unidos (IOM) publicó el informe: "Errar es humano"1 donde se advierte sobre la frecuencia y gravedad de los errores causados por profesionales de la salud. Más adelante, la Organización Mundial de la Salud (OMS) exhortó a sus países miembros sobre la necesidad de formular políticas y prácticas relacionadas con la seguridad del paciente e impulsar las investigaciones en esta temática ${ }^{2,3}$, basada en estudios que lo configuran como un grave problema de salud pública, pues se calcula que, en el caso de los países desarrollados, hasta uno de cada 10 pacientes sufre algún tipo de daño durante su permanencia en el hospital ${ }^{4}$.

Específicamente, los errores en la medicación son algunos de los eventos adversos más frecuentemente reportados en los estudios de prevalencia e incidencia en la mayoría de los países ${ }^{5,6}$. Por ejemplo, en el primer estudio Iberoamericano de eventos adversos (IBEAS) ${ }^{7}$, sobre incidentes que causan daño en la asistencia sanitaria, la medicación ocupó el segundo lugar tanto en prevalencia $(8,23 \%)$ como en incidencia $(9,87 \%)$. En la mayoría de los casos los errores relacionados con la utilización de medicamentos son multifactoriales, debido a que resultan de varios errores o fallos, presentes tanto en el sistema como los relacionados con los profesionales que están en contacto directo con los procesos, destacándose entre éstos el personal de enfermería ${ }^{8,9}$. Atendiendo a los lineamientos mundiales descritos, en Colombia se impulsa una política de seguridad del paciente ${ }^{10}$ y siendo la medicación uno de los procesos que con mayor frecuencia se registra como origen de eventos adversos, la presente investigación tuvo como objetivo indagar sobre las apreciaciones que tenía el personal de la salud en torno al grado de implementación del sistema de seguridad, concretamente en la utilización de medicamentos, en cada una de sus etapas: estandarización, almacenamiento, distribución, prescripción, administración y registro.

\section{Materiales y métodos}

Se realizó un estudio cuantitativo descriptivo en una población constituida por 73 trabajadores de la salud: 7 médicos $(9,6 \%), 13$ enfermeras $(17,8)$, 32 auxiliares de enfermería (43,8\%), 5 farmaceutas $(5,8 \%)$ y 16 auxiliares de farmacia $(21,9 \%)$, que se encontraban laborando en dos hospitales de segundo nivel del Departamento de Risaralda, durante los meses de octubre y noviembre del año. De esta manera, los resultados no pueden ser extrapolados a la población de instituciones de salud del departamento.

Se diseñó una encuesta a partir de dos instrumentos: el cuestionario de autoevaluación de la seguridad del sistema de utilización de los medicamentos en los hospitales, una adaptación del instituto para el uso seguro de los medicamentos (ISMP-España) ${ }^{10}$ y el cuestionario sobre seguridad de los pacientes: versión española del Hospital del Ministerio de Sanidad y Consumo ${ }^{11}$, contrastados con los lineamientos de la política de seguridad del paciente ${ }^{12}$ para 
Colombia. Se aplicó una prueba piloto al instrumento en una población similar de trabajadores de la salud previamente a la recolección de los datos.

Las preguntas indagaban sobre las apreciaciones de los trabajadores en torno al grado de cumplimiento institucional en aspectos generales de la política de seguridad del paciente y específicamente en la utilización de medicamentos en los procesos de estandarización, almacenamiento, distribución, prescripción, administración y registro.

Las respuestas se midieron mediante un baremo con cuatro categorías de respuesta: cumple, cumple medianamente, no cumple y no sabe. Se construyó una base de datos en el programa Excel que se exportó al programa SPSS, versión 16.0 para el análisis. Se calcularon porcentajes que se ordenaron de mayor a menor según la categoría "cumple" y se obtuvieron promedios de los porcentajes por cada categoría de respuestas y etapas del proceso de utilización de medicamentos.

\section{Consideraciones Éticas}

Según la Resolución 8430 de 1993 la investigación fue catalogada como sin riesgo y posterior a su aprobación por el Comité de Ética de la Universidad Libre se procedió a la aplicación del consentimiento informado y la encuesta a la población de estudio, que fue diligenciada por los informantes bajo dos modalidades: en forma dirigida por parte de los investigadores o de manera autónoma, previa explicación sobre su diligenciamiento.

\section{Resultados}

Se recolectaron un total de 73 encuestas en las dos instituciones, $58,9 \%$ en la primera y $41,1 \%$ en la segunda. El 9,6\% de los encuestados eran médicos, 17,8\% enfermeros, 43,8\% auxiliares de enfermería, 6,8\% regentes de farmacia y $21,9 \%$ auxiliares de farmacia. El $20,5 \%$ de los trabajadores tenían menos de 1 año de laborar en la institución, 42,5\% entre 1-5 años y el porcentaje restante $(37,0 \%)$ más de 5 años.

En cuanto al grado de aplicación de la política de seguridad del paciente en la institución, el porcentaje promedio de personas que declararon su total cumplimiento fue del $59,7 \%$ y el $29,3 \%$ dijeron que se cumplía medianamente (Tabla 1).

Tabla 1. Apreciaciones de los trabajadores frente al grado de cumplimiento institucional en aspectos generales de la política de seguridad del paciente en dos instituciones de salud de Risaralda, 2014

\begin{tabular}{|c|c|c|c|c|}
\hline Pregunta & No sabe & No cumple & $\begin{array}{l}\text { Cumple } \\
\text { mediana- } \\
\text { mente }\end{array}$ & Cumple \\
\hline $\begin{array}{l}\text { La institución tiene establecido un programa relacionado con la } \\
\text { seguridad del paciente }\end{array}$ & 5,5 & 0,0 & 15,1 & 79,5 \\
\hline $\begin{array}{l}\text { Las directivas de la institución han establecido un programa para la } \\
\text { reducción de errores en el proceso de administración de } \\
\text { medicamentos }\end{array}$ & 5,6 & 0,0 & 20,8 & 73,6 \\
\hline $\begin{array}{l}\text { Se educa, capacita, entrena y motiva al personal en la seguridad del } \\
\text { paciente }\end{array}$ & 4,3 & 0,0 & 31,4 & 64,3 \\
\hline $\begin{array}{l}\text { Existe coordinación entre las diferentes dependencias de la } \\
\text { institución en relación con la seguridad del paciente }\end{array}$ & 5,6 & 5,6 & 32,4 & 56,3 \\
\hline $\begin{array}{l}\text { Se garantiza la estabilidad del personal en pro de la seguridad del } \\
\text { paciente }\end{array}$ & 9,7 & 4,2 & 41,7 & 44,4 \\
\hline $\begin{array}{l}\text { El descanso del personal corresponde al volumen de trabajo } \\
\text { clínico, sin comprometer la seguridad del paciente }\end{array}$ & 17,1 & 8,6 & 34,3 & 40,0 \\
\hline Promedio & 8,0 & 3,1 & 29,3 & 59,7 \\
\hline
\end{tabular}


Más bajos en la categoría de pleno cumplimiento, fueron las relacionadas con el descanso del personal, de acuerdo al volumen de trabajo $(40,0 \%)$, su estabilidad $(44,4 \%)$ y la existencia de coordinación entre las diferentes dependencias de la institución (56,3\%).
Referente a las apreciaciones de los encuestados en la aplicación de la política, específicamente en la estandarización, almacenamiento y distribución de los medicamentos, el porcentaje promedio de personas que manifestaron un total cumplimiento fue del $65,1 \%$ (Tabla 2).

Tabla 2. Apreciaciones de los trabajadores en cuanto al grado de cumplimiento institucional en la estandarización, almacenamiento y distribución de los medicamentos

\begin{tabular}{|c|c|c|c|c|}
\hline Pregunta & No sabe & No cumple & $\begin{array}{c}\text { Cumple } \\
\text { mediana- } \\
\text { mente }\end{array}$ & Cumple \\
\hline La institución cuenta con químico farmacéutico & 10,3 & 7,4 & 4,4 & 77,9 \\
\hline $\begin{array}{l}\text { Se garantiza la cadena de frío para el almacenamiento y } \\
\text { dispensación de los medicamentos. }\end{array}$ & 5,6 & 2,8 & 5,6 & 86,1 \\
\hline $\begin{array}{l}\text { El contenido y el formato de etiquetado de los medicamentos se ha } \\
\text { estandarizado en la Institución con letra clara, nombre genérico, } \\
\text { lote y fecha de vencimiento. }\end{array}$ & 4,2 & 0 & 6,9 & 88,9 \\
\hline $\begin{array}{l}\text { En la farmacia se realiza el reempaque en dosis unitaria, empaque } \\
\text { de uno mayor a otro menor, debidamente identificado en su } \\
\text { etiqueta que contiene la dosis de un medicamento prescrito por el } \\
\text { médico, para ser administrado en atención domiciliaria u } \\
\text { hospitalaria. }\end{array}$ & 6,9 & 24,1 & 10,3 & 58,6 \\
\hline $\begin{array}{l}\text { El químico farmaceuta participa en los procesos de dispensación } \\
\text { de los medicamentos. }\end{array}$ & 20,3 & 4,7 & 20,3 & 54,7 \\
\hline $\begin{array}{l}\text { La institución cuenta con asesoría farmacológica por parte del } \\
\text { químico farmacéutico. }\end{array}$ & 16,2 & 7,4 & 23,5 & 52,9 \\
\hline $\begin{array}{l}\text { Se tiene protocolizado el uso racional de analgésicos en la } \\
\text { institución y se hace auditoria. }\end{array}$ & 13,9 & 12,5 & 22,2 & 51,4 \\
\hline $\begin{array}{l}\text { Al menos hay un funcionario farmacéutico de presencia física en el } \\
\text { centro de farmacia } 24 \text { horas al día, } 7 \text { días a la semana. }\end{array}$ & 9,1 & 27,3 & 13,6 & 50,0 \\
\hline Promedio & 10,8 & 10,8 & 13,4 & 65,1 \\
\hline
\end{tabular}

Los ítems que obtuvieron porcentajes más bajos para este componente fueron: la permanencia de un funcionario farmacéutico en la institución $(50,0 \%)$, la existencia de un protocolo para el uso racional de analgésicos en la institución y su respectiva auditoria $(51,4 \%)$, la existencia de asesoría farmacológica por parte del químico farmacéutico (52,9\%), la participación del químico farmaceuta en los procesos de dispensación de los medicamentos $(54,7 \%)$ y el empaquetado de los medicamentos en la farmacia, en dosis unitaria, de mayor a menor y debidamente identificados $(58,6 \%)$. Frente a la prescripción de medicamentos el porcentaje promedio de respuestas que reflejaron su total cumplimiento fue de $61,7 \%$ siendo los ítems con porcentajes más bajos: la ubicación de muestras médicas en los servicios $(21,1 \%)$, la obtención de una historia farmacoterapéutica de los pacientes hospitalizados cuando ingresaban (49,3\%), la existencia de una cultura de reporte de reacciones al Instituto Nacional de Vigilancia de Medicamentos y Alimentos (INVIMA) (51,4\%), la identificación fácil del peso del paciente para la prescripción de los medicamentos $(54,3 \%)$ y la utilización de prescripciones verbales solo en casos de emergencias y cuando se realizaban procedimientos estériles (59,7\%) (Tabla 3). 
Tabla 3. Apreciaciones de los trabajadores en cuanto al grado de cumplimiento institucional en la prescripción de medicamentos

\begin{tabular}{|c|c|c|c|c|}
\hline Pregunta & No sabe & No cumple & $\begin{array}{c}\text { Cumple } \\
\text { mediana- } \\
\text { mente }\end{array}$ & Cumple \\
\hline $\begin{array}{l}\text { La información básica del paciente: nombre y apellidos, número de } \\
\text { cama, número de historia clínica, es clara, oportuna y puede verse en } \\
\text { todas las prescripciones }\end{array}$ & 2,9 & 1,4 & 4,3 & 91,3 \\
\hline $\begin{array}{l}\text { Se tiene un sistema establecido por parte del equipo de salud, que } \\
\text { incluye avisos destacados para la identificación de los pacientes } \\
\text { alérgicos a medicamentos }\end{array}$ & 10,1 & 0,0 & 10,1 & 79,7 \\
\hline La tarjeta de medicamentos es fácil de entender, es amplia y legible & 4,5 & 3,0 & 18,2 & 74,2 \\
\hline $\begin{array}{l}\text { Las prescripciones médicas se realizan en forma clara teniendo en } \\
\text { cuenta: fecha de orden del medicamento, nombre y apellidos del } \\
\text { paciente, } \\
\text { nombre del medicamento, dosis y vía de administración }\end{array}$ & 4,2 & 4,2 & 20,8 & 70,8 \\
\hline $\begin{array}{l}\text { Las enfermeras confirman con chequeo las prescripciones médicas } \\
\text { antes de su administración }\end{array}$ & 6,0 & 1,5 & 25,4 & 67,2 \\
\hline Existe un programa de fármaco-vigilancia en la institución & 17,8 & 2,7 & 19,2 & 60,3 \\
\hline $\begin{array}{l}\text { Las prescripciones verbales solo se utilizan en: casos de } \\
\text { emergencias y cuando se realizan procedimientos estériles y en } \\
\text { orden verbal la prescripción se repite de nuevo al médico para } \\
\text { confirmarla }\end{array}$ & 7,5 & 3,0 & 29,9 & 59,7 \\
\hline $\begin{array}{l}\text { El peso del paciente se identifica fácilmente para la prescripción de } \\
\text { los medicamentos }\end{array}$ & 11,4 & 8,6 & 25,7 & 54,3 \\
\hline Se tiene cultura de reporte de reacciones al INVIMA & 23,6 & 2,8 & 22,2 & 51,4 \\
\hline $\begin{array}{l}\text { Se obtiene una historia farmacoterapéutica de los pacientes } \\
\text { hospitalizados cuando ingresan, que incluye los medicamentos } \\
\text { formulados y sin formular, vitaminas etc }\end{array}$ & 17,4 & 10,1 & 23,2 & 49,3 \\
\hline No se encuentran muestras médicas en los servicios & 26,3 & 29,8 & 22,8 & 21,1 \\
\hline Promedio & 12,0 & 6,1 & 20,2 & 61,7 \\
\hline
\end{tabular}

Fueron calificados con cumplimiento alto los siguientes ítems: la claridad, oportunidad y visibilidad de la información básica del paciente: nombre y apellidos, número de cama, número de historia clínica $(91,3 \%)$, la existencia de un sistema por parte del equipo de salud, que incluía avisos destacados para la identificación de los pacientes alérgicos a medicamentos $(79,7 \%)$, la claridad y legibilidad de la tarjeta de medicamentos $(74,2 \%)$ y la claridad de las prescripciones médicas $(70,8 \%)$. En relación con la administración y el registro de los medicamentos, el porcentaje promedio de trabajadores que indicaron cumplimiento de este componente fue del $65,5 \%$ y las respuestas que representaron una observancia menor fueron: la estandarización de las concentraciones de las soluciones para infusión de los medicamentos de alto riesgo $(35,8 \%)$, el establecimiento de un sistema para evitar el riesgo de producción de errores por la similitud de la presentación de los medicamentos $(43,5 \%)$ y por la similitud de su nombre $(44,8 \%)$, la supervisión de enfermería en la administración de medicamentos $(50,7 \%)$ y el fomento de la notificación de errores en la mira de establecer correctivos y planes de mejoramiento $(54,2 \%)$ (Tabla 4$)$. 
Tabla 4. Apreciaciones de los trabajadores en cuanto al grado de cumplimiento institucional en la administración y registro de medicamentos

\begin{tabular}{|c|c|c|c|c|}
\hline Pregunta & No sabe & No cumple & $\begin{array}{c}\text { Cumple } \\
\text { mediana- } \\
\text { mente }\end{array}$ & Cumple \\
\hline $\begin{array}{l}\text { Se controla la humedad relativa, la temperatura y se tienen los } \\
\text { registros en los cuartos de almacenamiento de los medicamentos }\end{array}$ & 4,1 & 0,0 & 8,2 & 87,7 \\
\hline $\begin{array}{l}\text { Los medicamentos se prescriben, transcriben, preparan, dispensan y } \\
\text { administran en un entorno físico con espacio e iluminación adecuados }\end{array}$ & 0 & 2,8 & 13,9 & 83,3 \\
\hline $\begin{array}{l}\text { La institución tiene guías y protocolos actualizados para la } \\
\text { administración de medicamentos }\end{array}$ & 8,7 & 0,0 & 8,7 & 82,6 \\
\hline $\begin{array}{l}\text { Existen procesos para el manejo adecuado de los residuos } \\
\text { cortopunzantes }\end{array}$ & 4,1 & 2,7 & 11,0 & 82,2 \\
\hline Se registran correctamente los medicamentos administrados & 7,4 & 1,5 & 14,7 & 76,5 \\
\hline $\begin{array}{l}\text { Los medicamentos se dispensan en los servicios de forma segura y } \\
\text { están disponibles para su administración dentro de los tiempos } \\
\text { apropiados para satisfacer las necesidades de los pacientes }\end{array}$ & 2,8 & 0,0 & 23,6 & 73,6 \\
\hline $\begin{array}{l}\text { Se ha estandarizado el horario de administración de los } \\
\text { medicamentos }\end{array}$ & 7,1 & 2,9 & 18,6 & 71,4 \\
\hline $\begin{array}{l}\text { Los productos químicos líquidos, incluyendo los compuestos de } \\
\text { limpieza, están etiquetados claramente en su composición y se } \\
\text { almacenan por separado de los medicamentos }\end{array}$ & 10,0 & 4,3 & 14,3 & 71,4 \\
\hline $\begin{array}{l}\text { El personal de enfermería conoce y aplica los } 10 \text { correctos en la } \\
\text { administración de medicamentos }\end{array}$ & 6,0 & 4,5 & 19,4 & 70,1 \\
\hline $\begin{array}{l}\text { Los registros de los medicamentos están sistematizados por medio } \\
\text { electrónico }\end{array}$ & 4,5 & 14,9 & 10,4 & 70,1 \\
\hline $\begin{array}{l}\text { Las hojas de medicamento de enfermería se llevan hasta la cama del } \\
\text { paciente para su confirmación }\end{array}$ & 13,6 & 1,5 & 16,7 & 68,2 \\
\hline $\begin{array}{l}\text { Los medicamentos de alto riesgo están definidos, identificados y han } \\
\text { sido comunicados a todos los funcionarios del equipo de salud que los } \\
\text { prescriben, dispensan y administran }\end{array}$ & 11,3 & 2,8 & 19,7 & 66,2 \\
\hline $\begin{array}{l}\text { Se utilizan prácticas eficaces para el control de infecciones cuando se } \\
\text { almacenan, preparan y administran medicamentos }\end{array}$ & 10,0 & 1,4 & 24,3 & 64,3 \\
\hline $\begin{array}{l}\text { La institución cuenta con una guía de reacción inmediata frente a } \\
\text { eventos adversos en relación con la administración de medicamentos }\end{array}$ & 13,9 & 4,2 & 18,1 & 63,9 \\
\hline $\begin{array}{l}\text { Se han establecido criterios para determinar cuándo se utilizan las } \\
\text { bombas de infusión }\end{array}$ & 12,1 & 7,6 & 21,2 & 59,1 \\
\hline $\begin{array}{l}\text { Se encuentra establecido un sistema para evitar el riesgo de errores } \\
\text { por la similitud del nombre y apellidos de los pacientes }\end{array}$ & 18,3 & 15,5 & 11,3 & 54,9 \\
\hline $\begin{array}{l}\text { Se fomenta la notificación y descripción de errores en la } \\
\text { administración de medicamentos entre el equipo de salud y un grupo } \\
\text { multidisciplinar, para realizar correctivos y planes de mejoramiento }\end{array}$ & 11,1 & 2,8 & 31,9 & 54,2 \\
\hline $\begin{array}{l}\text { Se realiza supervisión de enfermería en la administración de } \\
\text { medicamentos }\end{array}$ & 10,1 & 5,8 & 33,3 & 50,7 \\
\hline $\begin{array}{l}\text { Se encuentra establecido un sistema para evitar el riesgo de } \\
\text { producción de errores por la similitud del nombre de los } \\
\text { medicamentos }\end{array}$ & 20,9 & 9,0 & 25,4 & 44,8 \\
\hline $\begin{array}{l}\text { Se encuentra establecido un sistema para evitar el riesgo que se } \\
\text { produzcan errores por la similitud de la presentación de los } \\
\text { medicamentos }\end{array}$ & 14,5 & 17,4 & 24,6 & 43,5 \\
\hline $\begin{array}{l}\text { Las concentraciones de las soluciones para infusión de los } \\
\text { medicamentos de alto riesgo están estandarizadas en una } \\
\text { concentración única }\end{array}$ & 31,3 & 16,4 & 16,4 & 35,8 \\
\hline Promedio & 10,6 & 5,6 & 18,4 & 65,5 \\
\hline
\end{tabular}




\section{Discusión}

Las respuestas de los encuestados relacionadas con aspectos generales de la política de seguridad del paciente muestran que el cumplimiento en su aplicación es parcial, reflejando en algunas respuestas, situaciones de orden gerencial que dificultan su adecuado funcionamiento como son: el cambio continuo de personal, la no correspondencia entre el volumen de trabajo y el descanso del personal y la falta de coordinación entre las diferentes dependencias de la institución.

El sentimiento de falta de descanso del personal frente a un exceso de trabajo, manifestado por los trabajadores encuestados como causa de incumplimiento de los lineamientos de la política de seguridad del paciente, probablemente esté mediado por la insuficiencia de talento humano profesional en las instituciones. Estas carencias aunadas a la falta de coordinación entre servicios, también fueron identificadas como algunas de las mayores debilidades para garantizar la seguridad de los pacientes en un estudio realizado en una red de hospitales públicos de España ${ }^{13}$. Estudios similares ${ }^{14,15}$ argumentan también que la insuficiente vinculación de personal, es un factor relacionado con los incidentes de seguridad, denotando la necesidad de contratar mas personal y organizar sus ritmos de trabajo, como una posibilidad de mejorar la seguridad de los pacientes, afectada por la carga excesiva de trabajo de los profesionales.

Concretamente, sobre la percepción de los trabajadores en cuanto a los aspectos de seguridad en la utilización de medicamentos, los porcentajes de respuestas promedio de todas las etapas del proceso, demuestran que prácticamente la mitad de los encuestados aprecian deficiencias. Los eventos adversos relacionados con medicación suelen ser frecuentes de acuerdo con diversos estudios $7,16-18$ y la $\mathrm{OMS}^{19}$, refiere que según investigaciones, en los servicios de atención de casos agudos, entre un $7,0 \%$ y un $10,0 \%$ de los pacientes experimenta algún evento adverso relacionado con los medicamentos. De ellos, aproximadamente un $28,0 \%$ a un $56,0 \%$ son prevenibles. Los resultados del presente estudio, según las apreciaciones de los encuestados, señalan que evidentemente existen fallas en los sistemas de seguridad que garanticen unos procesos adecuados y confiables en la medicación de los pacientes, en las instituciones estudiadas.

Particularmente, en la estandarización, almacenamiento y distribución de los medicamentos, se obtuvieron bajos porcentajes referente a la permanencia de un químico farmacéutico en la institución y la asesoría farmacológica por parte de este profesional. Es importante tener en cuenta que su presencia contribuye a la seguridad en la atención del paciente y por lo tanto las instituciones deben garantizar su permanencia y participación activa para lograr la calidad de todos los procesos que impliquen la utilización de medicamentos, sin olvidar que la prevención de errores exige pensar en términos de sistema, es decir, precisa la participación y el esfuerzo de todos los implicados en la cadena terapéutica del medicamento ${ }^{20}$. En torno a la prescripción se señala por ejemplo, la baja calificación obtenida en el ítem sobre existencia de muestras médicas en los servicios de hospitalización, lo cual incrementa el riesgo de administración de medicamentos vencidos y limita el control de calidad y su control por cada paciente.

Es también preocupante el bajo cumplimiento, según los encuestados, en la estandarización de las concentraciones de las soluciones para infusión de los medicamentos de alto riesgo ${ }^{21}$, situación que se agrava considerando la similar apreciación de los trabajadores sobre la existencia de una guía de reacción inmediata frente a eventos adversos. En un estudio sobre incidencia de reacciones adversas a medicamentos en un hospital de alta complejidad en Colombia, el $34,6 \%$ de 2.973 reportes, fue por medicinas de alto riesgo y aproximadamente la mitad de éstos eran evitables $^{22}$, evidenciando que es posible minimizar los errores si se tiene estandarizado su manejo. 
En una revisión de publicaciones enfocadas a la administración de medicamentos en la atención de enfermería, en el periodo 2007 a 2011, se encontró que el $25,0 \%$ de los errores ocurridos estaban relacionados con la falta de interacción entre el equipo multidisciplinar y la falta de conocimiento suficiente para resolver cualquier impase a la hora de administrar los medicamentos ${ }^{23}$. Estudios han reportado que el $30,2 \%$ de los profesionales de enfermería no definía correctamente la reacción adversa ${ }^{24}$. Si bien en la presente investigación no se indagó por el conocimiento del personal de enfermería para detectar y enfrentar reacciones adversas, de acuerdo con las cifras presentadas, es necesario considerarlo a la hora de implementar una política de seguridad del paciente, con el fin de desarrollar procesos de capacitación y actualización permanente de estos profesionales.

Por su parte, la percepción negativa frente a la existencia de sistemas para evitar el riesgo que se produzcan errores por la similitud de la presentación de los medicamentos, o de sus nombres, está indicando que estas instituciones no cuentan con acciones preventivas de este tipo, que busquen minimizar los riesgos que comprometen la seguridad de los pacientes, como lo evidenció un estudio realizado en seis hospitales brasileños en el $2010^{25}$ pudo apreciarse que por cada 1000 medicamentos administrados, 17 fueron diferentes a los prescritos por el médico y en otro similar, la droga incorrecta fue identificada como una causa importante de error ${ }^{26,27}$. En consecuencia, el no poseer sistemas que reduzcan la posibilidad de falla humana, puede afectar la seguridad de los pacientes, incluso con resultados fatales.

Es necesario considerar que según los encuestados existe pobre cumplimiento, según los encuestados, del ítem sobre la existencia de un programa de fármaco-vigilancia, toda vez que esta disciplina es fundamental para la detección, evaluación, conocimiento y prevención de reacciones adversas y otros posibles problemas relacionados con los medicamentos ${ }^{28}$. Para lograrlo, es necesario generar la cultura del reporte institucional de reacciones adversas, con el fin de detectar las fallas o errores implicados e implementar los correctivos oportuna y adecuadamente. Las notificaciones de reacciones adversas son importantes no sólo para medir su incidencia real sino también para describir sus características en aras de obtener evidencias que generen decisiones adecuadas con respecto al medicamento ${ }^{29,30}$.

En cuanto a la percepción negativa de los trabajadores en torno a la existencia de prácticas eficaces para el control de infecciones cuando se almacenan, preparan y administran medicamentos, es necesario relacionarlo con el cumplimiento bajo que se obtuvo en el lavado de manos y el seguimiento de los cinco momentos de este procedimiento. Resultados similares fueron encontrados en tres instituciones de salud del departamento del Atlántico, donde se relata que éstas prácticas son conocidas y que están descritas en los protocolos, sin embargo hay incumplimiento en su aplicación ${ }^{31}$.

Lo anterior significa que todos los procesos que se relacionen con la seguridad del paciente en la administración de medicamentos deben ir acompañados de un monitoreo constante que garantice la aplicación de los protocolos existentes, máxime cuando el ítem sobre la existencia de supervisión permanente por parte de Enfermería tuvo una calificación baja, según las apreciaciones de los trabajadores.

Por último, es importante destacar los aspectos que tuvieron una buena calificación como fueron: la existencia de procesos para el manejo adecuado de los residuos corto-punzantes, de guías y protocolos actualizados para la administración de medicamentos, el entorno físico con espacio e iluminación adecuados, la garantía de la cadena de frío y el control y registro en los cuartos de almacenamiento. Además, la estandarización del etiquetado de los medicamentos en la institución con letra clara, nombre genérico, lote y fecha de vencimiento y la claridad y oportunidad de la información básica del paciente en todas las prescripciones. Esto último se contrapone a otros estudios donde las fuentes de error percibidas con mayor frecuencia fueron la transcripción y la legibilidad de las prescripciones manuscritas ${ }^{32-34}$. 


\section{Conclusiones}

Se evidenció que aproximadamente la mitad de los encuestados aprecian falta de cumplimiento en la implementación de la política de seguridad del paciente que enmarca los procesos de utilización de medicamentos, en todas sus etapas: estandarización, almacenamiento, distribución, prescripción, administración y registro.

Los trabajadores encuestados perciben situaciones de orden gerencial que afectan la implementación general de la política dificultan su adecuado funcionamiento como son: el cambio continuo de personal, la no correspondencia entre el volumen de trabajo y el descanso del personal y la falta de coordinación entre las diferentes dependencias de la institución.

Enfermería juega un rol de máxima importancia en la prevención de errores de medicación, pero es sólo un eslabón en la cadena terapéutica del medicamento que amerita decisiones administrativas y gerenciales en un contexto institucional que exige la participación y el esfuerzo de todos los implicados.

\section{Agradecimientos}

Las autoras expresan sus agradecimientos a los estudiantes del Programa de Enfermería de la Universidad Libre: Sebastián Betancurth Ramírez, Alejandra María Usma López, Juan Manuel Sisco Pancha, Claudia Viviana Peña Ramírez y Viviana Andrea Loaiza Escobar, por su aporte significativo a este trabajo.

Conflicto de intereses: Ninguno declarado.

\section{Referencias}

1. Kohn LT, Corrigan JM, Donaldson MS, Eds. To err is human: Building a safer health system. Committee on Health Care in America. Institute of Medicine. Washington DC: National Academy Press; 1999.

2. OMS. La Organización Mundial de la Salud y sus asociados instan a una intensificación de las investigaciones para mejorar la seguridad del paciente. [Consultado septiembre 3 de 2015] 2007. Disponible en: http://www.who.int/mediacentre/news/releases/200 7/pr52/es/.

3. OMS. Marco Conceptual de la Clasificación Internacional para la Seguridad del Paciente Versión 1.1, informe técnico definitivo; enero de 2009 [citado septiembre 3 de 2015] Disponible en: http://www.who.int/patientsafety/implementation/ic ps/icps_full_report_es.pdf.

4. OMS. 10 datos sobre seguridad del paciente. Junio 2014 [citado septiembre 3 de 2015] Disponible en: http://www.who.int/features/factfiles/patient_safety/ es/.

5. Lima FDSS, Souza NPG, Freire de Vasconcelos P, Aires de Freitas CH, Bessa-Jorge MS, De Souza-Oliveira AC. Implicaciones de la seguridad del paciente en la práctica del cuidado de enfermería. Enfermería Global. 2014;13(3):293-309.

6. Plaza JA. Reto en seguridad del paciente: Reducir los EA más de un 50\%. Diario Médico. 2015. [citado septiembre 3 de 2015] Disponible en: http://search.proquest.com/docview/1705787431?ac countid=46889

7. Ministerio de Sanidad y Política Social. Estudio IBEAS: prevalencia de efectos adversos en hospitales de Latinoamérica. [citado septiembre 3 de 2015] Disponible https://www.minsalud.gov.co/sites/rid/Lists/Bibliote caDigital/ RIDE/ DE/ CA/ RESULTADOS\%20ESTUDI0\%20IBEAS.pdf.

8. Bond C, Raehl C, Franke T. Clinical Pharmacy Services, Hospital Pharmacy Staffing, and Medication Errors in the United States Hospitals. Pharmacotherapy. 2002;22(2):134-47.

9. Hepler C. Regulating for outcomes as a systems response to the problem of drug-related morbidity. J Am Pharm Assoc. 2001;41(1):108-15.

10. Instituto para el uso seguro de los medicamentos. Cuestionario de autoevaluación de la seguridad del sistema de utilización de los medicamentos en los hospitales. Madrid: Ministerio de Sanidad y consumo. S.F. [citado enero 25 de 2016] Disponible en: http://www.ismpespana.org/ficheros/cuestionario.pdf.

11. España, Ministerio de Sanidad y Consumo. Cuestionario sobre seguridad de los pacientes: versión española del Hospital Survey on Patient Safety. Madrid: Ministerio de Sanidad y Consumo; 2005.

12. Colombia, Ministerio de la Protección social. Lineamientos para la implementación de la política de seguridad del paciente; Nov 2008. [citado septiembre 3 de 2015] Disponible en: http://www.acreditacionensalud.org.co/catalogo/docs /Lineamientos\%20politica\%20seguridad\%20paciente. pdf.

13. Da Silva ZA, De Souza AC, Saturno PJ. Cultura de seguridad del paciente y factores asociados en una red de hospitales públicos españoles. Cad. Saúde Pública. 2013;29(2):283-293

14. Hellings J, Schrooten W, Klazinga N, Vleugels A. Challenging patient safety culture: survey results. Int J Health Care Qual Assur. 2007;20:620-32. 
15. Kho ME, Carbone JM, Lucas J, Cook DJ. Safety climate survey: reliability of results from a multicenter ICU survey. Qual Saf Health Care. 2005;14:273-8.

16. Aranaz J, Aibar C, Limón R, Mira JJ, Vitaller J, Agra Y, Terol E. A study of the prevalence of adverse events in primary healthcare in Spain. Eur J Public Health. 2012; 22(6):921-5.

17. Lancis-Sepúlveda ML, Asenjo-Araya C. Estudio de incidencia de eventos adversos en una clínica privada en Chile. Rev Calidad Asistencial.2014;29(2):78-83

18. Silva AEBC, Reis AMM, Miasso AI, Santos JO, Cassiani SHB. Adverse drug events in a sentinel hospital in the State of Goiás, Brazil. Rev. Latino-Am. Enfermagem. 2011;19(2):378-86.

19. Organización Mundial de la Salud. Eventos adversos relacionados con medicamentos. [citado marzo 12 de 2017] Disponible en: http://www.who.int/patientsafety/information_centre /documents/ps_research_brochure_es.pdf?ua=1

20. Otero MJ. El papel del farmacéutico en la prevención de los errores de medicación En: Fundación Promedic (eds.) Educación continuada para farmacéuticos de hospital II. s.f. [citado enero 16 de 2016] Disponible en:

http://www.ismp-espana.org/ficheros/Fichero17.pdf

21. Institute for Safe Medications Practices. ISMP's list of high alert medications [monografía en Internet]. Horsham, PA: ISMP; 2013 [citado 2013 Feb 28]. Disponible en: http://www.ismp.org.

22. Botero JP, Restrepo AM. Caracterización de las reacciones adversas a medicamentos reportadas al programa de fármaco-vigilancia de un hospital de alta complejidad $\mathrm{y}$ factores asociados con su evitabilidad. Medicina UPB. 2013;32(2):129-137.

23. Silva RP, Barreto BMF, Tenorio DM, Camacho ACLF, De Oliveira B, Guitton B. Revisión integradora acerca de administración de medicamentos en la atención de enfermería. Revista De Pesquisa, Cuidado é Fundamental Online. 2013;5(5):36-44.

24. Felipe AOB, de Oliveira MB, Terra F. Conocimiento del equipo de enfermería que trabaja en unidades pediátrica y neonatal sobre reacciones adversas a medicamentos. Enfermería Global. 2014;13(3):1-10.

25. De Bortoli SHC, Silvia AAM, Bauer SAE, Trevisani FF, Perufo SO, Alux TTC. Identificación y análisis de los errores de medicación en seis hospitales brasileños. Cienc enferm. 2010;16(1):85-95.

26. Franco JN, Ribeiro G, D' Innocenzo M, Barros BPA. Perception of de nursing team about causes of errors in the administration of medication. Rev Bras Enferm. 2010 nov-dic; 63(6):927-932.

27. Toffoletto MC, Juan M, Arce DM, Guerra AO, Rodríguez CAV. Errors in the preparation and administration of medications: An integrative review of latin american literatura. Enfermería Global. 2015;14(1):361-371.

28. Virga C, Aguzzi A. Farmaco-vigilancia: estudio de las reacciones adversas a medicamentos (RAM). Archivos venezolanos de farmacología y terapéutica. 2011;30(3):61-63.

29. Pushkin R, Frassetto L, Tsourounis C, Segal EL, Kim S. Improving the reporting of adverse drug reactions in the hospital setting. Postgrad Med. 2010;122(6):15464.

30. Wooten JM. Adverse drug reactions: part II. South Med J. 2010;103(11):1138-45.

31. Caro SE, Díaz D, De las Salas R, Gutiérrez E, Lemus C, Quintero S. Conocimientos relacionados con aspectos de la administración de medicamentos en la práctica de enfermería en tres hospitales del Atlántico (Colombia). Salud Uninorte. 2014; 30(3):371-380.

32. Gómez OJ, Soto A, Arenas A, Garzón J; González A. Una mirada actual de la cultura de seguridad del paciente. Avances en Enfermería. 2011;XXIX(2):363-374.

33. Armutlu M, Foley ML Surette J, Belzile E, McCusker J. Survey of nursing perceptions of medication administration practices, perceived sources of errors and reporting behaviours. Healthcare Quarterly. 2008; 11(Special Issue):1114-1123.

34. Gimenes F, Miasso A, Junior L, Grou C. Prescripción informatizada como factor contribuyente para la seguridad de los pacientes hospitalizados. Pharmacy pract. 2006;4(1):13-17. 